\title{
Supraventricular tachycardia as the initial presentation of bacterial infective endocarditis: A rare case report
}

\author{
Wei-Tsung $\mathrm{Wu}^{1}$, Hung-Ling Huang ${ }^{2}$, Ho-Ming Su${ }^{1,3}$, Tsung-Hsien Lin ${ }^{1,3}$, Kun-Tai Lee ${ }^{1,3}$, \\ Sheng-Hsiung Sheu ${ }^{1,3}$, Po-Chao Hsu ${ }^{1,3}$
}

1. Division of Cardiology, Department of Internal Medicine, Kaohsiung Medical University Hospital, Kaohsiung Medical University, Kaohsiung, Taiwan, ROC. 2. Division of Pulmonary and Critical Care Medicine, Department of I nternal Medicine, Kaohsiung Medical University Hospital, Kaohsiung Medical University, Kaohsiung, Taiwan, ROC. 3. Department of Internal Medicine, Faculty of Medicine, School of Medicine, Kaohsiung Medical University, Kaohsiung, Taiwan, ROC

Correspondence: Po-Chao Hsu. Address: Division of Cardiology, Department of Internal Medicine, Kaohsiung Medical University Hospital, 100 Tzyou 1st Road, Kaohsiung. 80708, Taiwan, ROC. Email: pochao.hsu@gmail.com

Received: May 19, 2015

DOI : $10.5430 /$ crim.v2n3p26
Accepted: June 15, 2015

URL: http://dx.doi.org/10.5430/crim.v2n3p26

\section{Abstract}

Infective endocarditis (IE) is an infection of the heart valves or the heart's inner lining. The clinical symptoms of infectious endocarditis vary considerably, some are subtle and non-specific, which make the diagnosis difficult or the signs misleading. In some cases cardiac symptoms are associated with intra cardiac extension of the infection, including murmur, conduction blocks and congestive heart failure. However, there was no literature description of supraventricular tachycardia (SVT) as the initial presentation of IE. Herein we report a case of bacterial IE with SVT as the initial presentation of disease, which finally leaded to catastrophic outcome. This rare case should be the reminder for physicians that in patients with SVT in combination with higher level of inflammatory markers, one should also consider the possibility of IE, to help to avoid its catastrophic consequences.

\section{Keywords}

Supraventricular tachycardia, Infective endocarditis

\section{I ntroduction}

Infective endocarditis (IE) is defined as an infection of the endocardial surface of the heart, which may involve one or more heart valves, the mural endocardium or a septal defect. It has an estimated annual incidence of 3 to 9 cases per 100,000 persons in industrialized countries, and occurs in male predominantly ${ }^{[1,2]}$. The clinical presentation of IE varies from patient to patient. Fever occurs in $97 \%$ of people, other symptoms include fatigue, shortness of breath, anorexia, headache, myalgias, joint pain. The role of electrocardiography (ECG) in recognizing IE is controversial. Non-specific changes in ECG are quite common in endocarditis and in some cases indicate a life-threatening complication, thereby predicting high morbidity and mortality. Sinus tachycardia is the most common ECG finding in previous literature ${ }^{[3-5]}$, however, supraventricular tachycardia (SVT) was rarely reported in IE patients. Herein, we presented a rare case without typical clinical symptoms of bacterial IE, but SVT as the initial presentation. Due to the nonspecific symptoms, IE diagnosis was delayed what finally led to the catastrophic outcome. 


\section{Case presentation}

A 59-year-old male suffered from persistent palpitation was brought to the emergency department (ED) for help. Associated symptoms included chest discomfort, diaphoresis, shortness of breath and fainting feeling. Tracing back his history, the patient was a smoker, with a past medical history of type 2 diabetes mellitus and hypertension, but denied any previous heart disease or cardiac arrhythmias. In addition, he also denied taking intravenous drugs.

At the admission heart rate was 180 beats $/ \mathrm{min}$, blood pressure $114 / 53 \mathrm{mmHg}$ and temperature $36.8^{\circ} \mathrm{C}$. Physical examination revealed a Grade 2/6 pansystolic murmur over apex and left sternal area. There were no other specific infectious symptoms/signs noted by history taking and physical examination. Laboratory investigations revealed leukocytosis with neutrophil predominant (white blood cell: 20900/ul, neutrophil: 89\%), C-reactive protein (CRP): $53 \mathrm{mg} / \mathrm{L}$, B-type natriuretic peptides (BNP): $700 \mathrm{pg} / \mathrm{ml}$. Other parameters such as renal function, liver function and electrolytes were in normal value range. Initial ECG revealed SVT with 179 beats/min (see Figure A). Chest X-ray (CXR) showed normal finding. In order to relieve the symptoms of palpitation and chest discomfort, two doses of adenosine (6 mg) were given intravenously and SVT was converted to the normal sinus rhythm. After initial management, the patient felt relief in symptoms and then he was referred to cardiovascular outpatient department for further survey and treatment.

A serial of examinations were arranged for arrhythmia and heart murmurs diagnosis. 24 hour Holter ECG revealed sinus rhythm with intermittent SVTs (ventricular rate 120 189/min), frequent atrial premature complexes, and some multiform bigeminy, trigeminy and pairs of ventricular premature complexes (see Figure B).Transthoracic echocardiography showed a movable vegetation (size: $1.2 \mathrm{~cm} \times 1.0 \mathrm{~cm}$ ) over anterior mitral leaflet (see Figure C \& D) with moderate mitral regurgitation and preserved left ventricular ejection fraction (LVEF). According to echocardiographic findings and previous elevated inflammatory markers, the diagnosis of infective endocarditis was initially set. The patient was then admitted to our cardiovascular ward for further care.
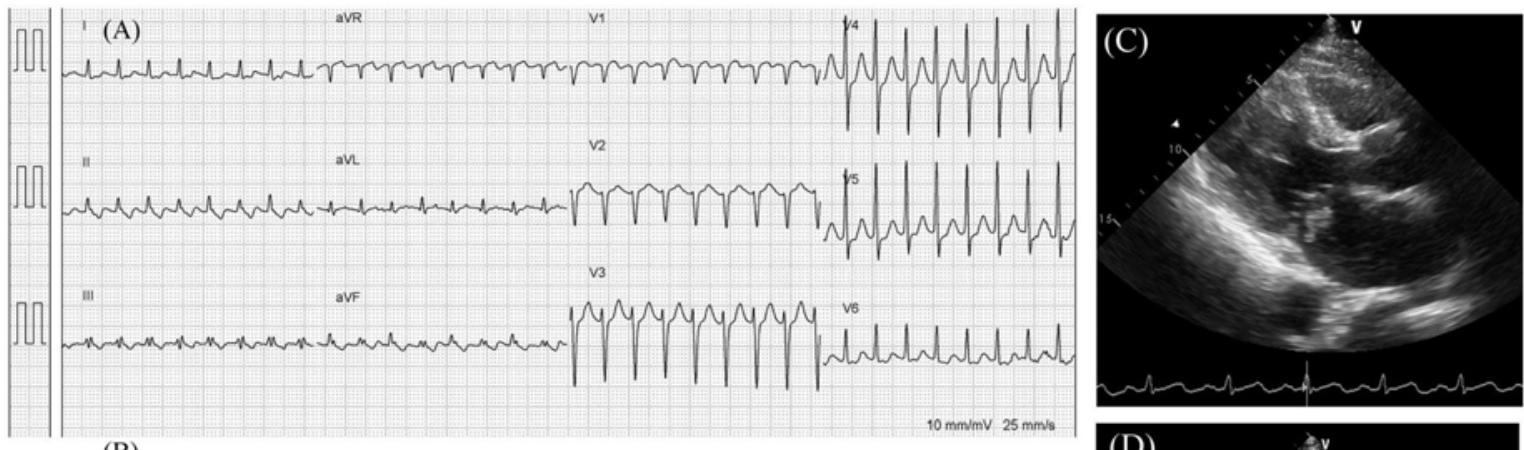

(B)
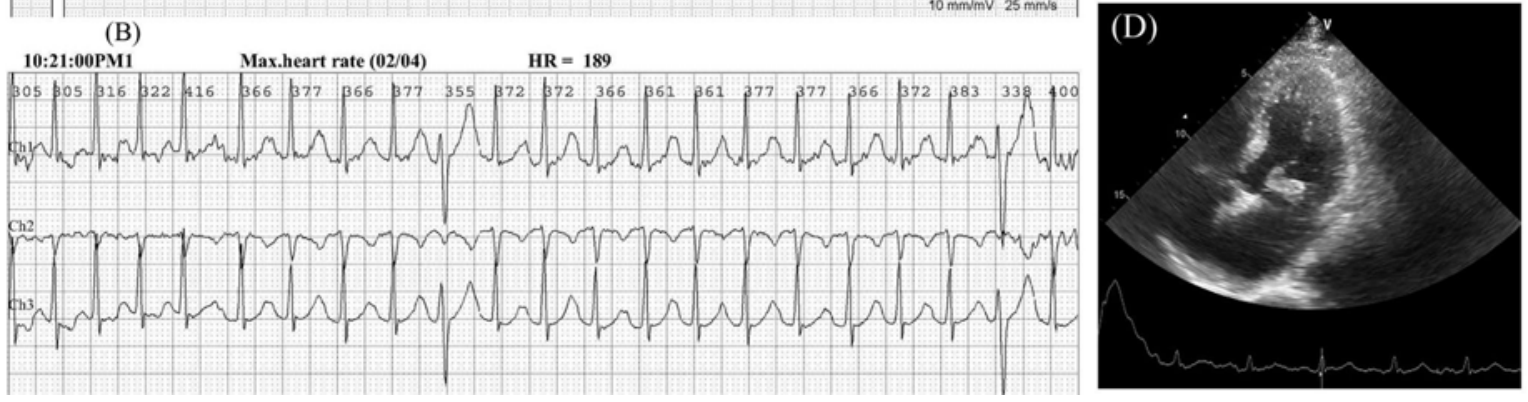

Figure. A: Initial ECG revealed supraventricular tachycardia (SVT); B: 24 hour Holter ECG revealed sinus rhythm with intermittent SVTs (ventricular rate 100 189/min); C \& D: Transthoracic echocardiography showed a movable vegetation $($ size: $1.2 \mathrm{~cm} \times 1.0 \mathrm{~cm}$ ) over anterior mitral leaflet from parasternal long axis view (C) and four chamber view (D)

Intravenous vancomycin was put into use for IE treatment. However, on the third day of admission, the patient had progressive dyspnea, orthopnea and chest tightness, which later led to desaturation. No serial change of cardiac enzymes was noted. CXR revealed enlarged of hilar vascular shadow and patchy infiltration with air-bronchogram appearance over 
bilateral lung field, which was compatible with pulmonary edema and superimposed pneumonia with pleural effusion. The patient was transferred to our intensive care unit (ICU) and was intubated under ventilatory support for respiratory failure. The following laboratory data revealed increased inflammatory markers (white blood cell: 81,800/ul with neutrophil: 88\%, CRP: $255 \mathrm{mg} / \mathrm{L}$ ) and BNP: 3418 pg/ml. In addition to Vancomycin use, we further prescribed Tazocin $4.5 \mathrm{~g} 3$ times daily for nosocomial pathogens coverage. However, the clinical condition deteriorated quickly with progression to profound shock status. Central venous catheter was inserted for hemodynamic monitor. Initial central venous pressure level was $16 \mathrm{mmHg}$ and dopamine infusion was given for shock status and hemodynamic support. Continuous venovenous hemofiltration (CVVH) was then administered for anuria, metabolic acidosis and pulmonary edema. Follow-up echocardiography revealed deterioration of LVEF $(75 \% \rightarrow 43 \%)$ with severe mitral regurgitation. Four sets of blood cultures grew Streptococcus anginosus after 2 days of ICU admission. Cardiovascular surgeon was asked for evaluation of potential heart operation, but surgery was not favorable due to unstable hemodynamic status.

On the $8^{\text {th }}$ day of ICU stay, the CXR rapidly progressed to bilateral diffuse infiltration accompanied with sever hypoxemia $\left(\mathrm{PaO}_{2} / \mathrm{FiO}_{2}\right.$ ratio $<200 \mathrm{mmHg}$ ). Acute respiratory distress syndrome (ARDS) was recognized. We adjusted the ventilator setting to low tidal volume and high positive end-expiratory pressure and also titrated up the antibiotics to Meropenem with Teicoplanin; however, his condition still further deteriorated and finally expired after 18 days of intensive care.

\section{Discussion}

IE is a microbial infection of the endocardial surface of the heart. It occurred in most patients with prosthetic valves, intracardiac devices, or unrepaired cyanotic congenital heart diseases. Other risk factors include chronic rheumatic heart disease, age-related degenerative valvular lesions, hemodialysis and some coexisting conditions, such as diabetes, human immunodeficiency virus infection, and intravenous drug use ${ }^{[1,2]}$. According to the clinical course, it can divide into acute IE and subacute IE. The symptoms of early subacute native valve endocarditis are usually subtle and nonspecific, including low-grade fever, anorexia, weight loss, influenza-like syndromes, polymyalgia-like syndromes, pleuritic pain, syndromes similar to rheumatic fever, and abdominal symptoms ${ }^{[1,2]}$. To our knowledge, there was no literature revealing cardiac arrhythmia such as SVT as the initial presentation of IE and our case could be the first case with this rare finding. However, this atypical presentation seems not to be crucial for IE evaluation and may delay the diagnosis and appropriate therapy. As we know, IE remains a diagnostic and therapeutic challenge. Its manifestations may be muted by the indiscriminate use of anti bioticsor by underlying conditions in frail and elderly individuals or immunosuppressed people. The diagnosis of IE requires the integration of clinical, laboratory, and echocardiographic data. In general, the Duke criteria are clinically useful to establish the diagnosis of endocarditis ${ }^{[6]}$. However a modified version of the Duke criteria was proposed to cover the broader categorization of the possible cases, which based on misclassification of culture-negative cases, increasing role of trans esophageal echo cardiography and the relative risk of endocarditis in Staphylococcus aureus bacteremia ${ }^{[7]}$.

ECG is an effective, non-invasive tool to record the electrical activity of the heart and used to measure the heart's electrical conduction system. It provides clinical information to interpret it in the context of the symptoms/signs which the patients present. The manifestation of ECG changes is common in endocarditis, but there are no prospective studies in evaluating the electrocardiographic changes in those patients. ECG changes are common in endocarditis and indicate invasive disease, thereby predicting high morbidity and mortality ${ }^{[1]}$. Sinus tachycardia is the most common presentation in approximately 53\% of patients from autopsy study. Other observed findings are low QRS voltage, varying degree of AV block, ST elevation, and ventricular tachycardia ${ }^{[3-5]}$. There was only one case with SVT in the autopsy study and was not presented as the initial sign of IE as in presented case ${ }^{[5]}$. SVT is a series of rapid heartbeats that begin in or involve the upper chambers of the heart. It can cause the heart to beat very rapidly or erratically. As a result, the heart may beat inefficiently and further cause the patient to develop heart failure. 
The possible cause of abnormalities of rhythm or conduction disturbances may be interpreted variously as an effect of fever, intoxication or as a compensatory mechanism to the impaired contractile power of the heart ${ }^{[3]}$. In addition, the conduction abnormalities can also suggest the perivalvular region involvement and may indicate invasive disease, which is considered as the most possible mechanism of SVT in our case, and may predict high morbidity and mortality in these cases $^{[3,4]}$.

In conclusion, we presented a case of bacterial IE having SVT as the initial presentation and finally led to catastrophic outcome. This rare case reminds physicians that for patients with SVT in combination with higher inflammatory markers, we should not ignore the possibility of cardiac origin infection. Further detailed cardiac investigation should be performed to rule out the possible atypical infection such as IE to avoid catastrophic prognosis in these rare cases.

\section{Conflict of interests}

None declared.

\section{References}

[1] Hoen B, Duval X. Infective endocarditis. N Engl J Med. 2013; 368: 1425-33. PMid:23574121 http://dx.doi.org/10.1056/NEJMcp1206782

[2] Mylonakis E, Calderwood SB. Infective endocarditis in adults. N Engl J Med. 2001; 345: 1318-30. PMid:11794152 http://dx.doi.org/ 10.1056/NEJMra010082

[3] Meine TJ, Nettles RE, Anderson DJ, et al. Cardiac conduction abnormalities in endocarditis defined by the Duke criteria. Am Heart J. 2001; 142: 280-5. PMid:11479467 http://dx.doi.org/10.1067/mhj.2001.116964

[4] Nalmas S, Nagarakanti R, Slim J, et al. Electrocardiographic changes in infectious diseases. Hospital physician. $2007 ; 43: 15$.

[5] Berk WA. Electrocardiographic findings in infective endocarditis. J Emerg Med. 1988; 6: 129-32. PMid:3385174 http://dx.doi.org/10.1016/0736-4679(88)90153-9

[6] Durack DT, Lukes AS, Bright DK, et al. New criteria for diagnosis of infective endocarditis: utilization of specific echocardiographic findings. Am J Med. 1994; 96: 200-9. PMid:8154507 http://dx.doi.org/10.1016/0002-9343(94)90143-0

[7] Li JS, Sexton DJ, Mick N, et al. Proposed modifications to the Duke criteria for the diagnosis of infective endocarditis. Clin Infect Dis. 2000; 30: 633-8. PMid:10770721 http://dx.doi.org/10.1086/313753 